\title{
Testing a New Electron Microprobe and Developing New Analytical Protocols
}

\author{
Julien M. Allaz ${ }^{1}$
}

1. University of Colorado Boulder, Dept. of Geological Sciences, Boulder, CO (USA).

Since the development of the first electron probe microanalyzer (EPMA) [1], hardware and software have evolved considerably. Nowadays, it is possible to achieve high stability, precision and accuracy. Field emission (FE-) EPMA opens the door to sub-micron analysis at low (over-) voltage, the large-area monochromator and higher stability permit trace element analysis down to 1-10 ppm range, and Energy Dispersive Spectrometers (EDS) enable fast mineral identification, hyperspectral mapping, and good (standard-based) quantitative work. As the precision and stability of the instrument improve, more sensitive tests must be developed to ensure high quality analysis. This paper is an overview of the optimization and quality control of a new EPMA, and discusses the development of new analytical protocols, focusing on trace element analysis.

When setting up a new EPMA laboratory, the hardware setup is chosen based on what analysis types will be performed on a regular basis, allowing for enough versatility for unexpected analytical service requests. The choice of electron gun, spectrometers, and monochromators is crucial. On W or LaB 6 EPMA, wavelength dispersive spectrometers (WDS) are mostly equipped with TAP, PET and LiF monochromators, typically two to three of each, to permit analysis of energy X-ray lines between 0.5 and $12 \mathrm{keV}$. Depending on need, multilayer pseudocrystals of large 2d spacing (LDEx, PCx) are installed to permit low energy X-ray analysis. On FE-EPMA, the optimum working conditions for higher spatial resolution are around 5-10 keV, which require more large-2d pseudocrystals or a Soft X-ray Emission Spectrometer (SXES). All monochromators are available as large-area crystals, which increase the count rate 2- to 3-fold, and even more with JEOL's H-type spectrometer (100 mm Rowland circle) or Cameca's very-large PET crystals, although at the cost of more limited spectrometer motion. EDS, panchromatic or monochromatic cathodoluminescence detectors, cold fingers, anticontamination systems, and other devices can complete the EPMA. The JEOL-8230 installed at CU Boulder in 2016 is equipped with a $\mathrm{LaB}_{6}$ electron gun, a $10 \mathrm{~mm}^{2}$ SDD EDS detector, a panchromatic CL detector, and has been optimized for trace element analysis with four spectrometers equipped with large-area monochromators.

Once the instrument is installed, it is desirable to develop procedures that confirm the performance of the EPMA with regards to imaging and qualitative or quantitative analysis, and to demonstrate the precision and accuracy of data collected [2]. EPMA designers have their own series of standardized tests to ensure data quality and instrument reliability. Whereas these tests are necessary, they are often insufficient and should be supplemented with tests adapted to the type of analyses to be performed in the laboratory [3, 4]. To guarantee analysis quality, quality control tests should be performed at installation and repeated regularly. These tests should cover all crucial instrument parts: column, stage, and all detectors. WDS deserves special attention, and all of its components should be carefully checked (step motor \& gears, spectrometer alignment, PHA and X-ray counters, etc.) to ensure optimum conditions.

Once the instrument performance is confirmed, an analytical protocol can be developed. Ideally, the list of elements to be analyzed is known, as is the expected concentration level. Otherwise, a 1-hour full WDS scan will help to assess the presence of most constituents down to $100-500$ ppm range (15 to $25 \mathrm{keV} ; 100$ to $200 \mathrm{nA} ; 1.5^{*} 10^{-4} \sin \theta$ step; $1.5 \mathrm{sec}$ per point). WDS scans are also recommended for complex materials 
to assess potential background and peak interferences, along with background curvature. With a list of elements defined, the optimum spectrometer and column settings can be chosen. If spatial resolution is of concern, a Monte-Carlo simulation (e.g., Casino [5] or similar) can give an estimate of the analytical volume. For trace elements analysis, the total counts and thus the precision is increased by (a) using largearea monochromators, (b) aggregating intensities from multiple spectrometers, (c) optimizing the acceleration voltage and the PHA, and (d) increasing the current and the counting time. Beam damage is more likely at high current and long counting time, and beam stability tests should be run to avoid inaccurate results. Defocusing the beam, using different coating materials (metals; e.g., Al, Ag, Ir, Os), and/or combining major element analysis on EDS can all potentially help to reduce inaccuracies resulting from beam damage. By using the Mean Atomic Number background correction (MAN; [6]), the total analysis time can be cut in half, as no background is acquired on the unknown. The use of a time-dependent intensity (TDI) correction can counteract the change in count rate over time, at least for the first elements analyzed on each spectrometer.

Among different analytical setups developed over recent months in my laboratory, the one regarding REEbearing minerals was probably the most challenging. For instance, work on monazite $\mathrm{U}-\mathrm{Th}-\mathrm{Pb} \mathrm{btal}_{\text {tal }}$ dating by EPMA ideally requires both a complete chemical analysis and a precise and accurate measurement of $\mathrm{Th}, \mathrm{U}$, and (trace amount of) $\mathrm{Pb}$. A fast first setup was designed using the MAN background correction to analyze as many elements as possible (35 so far) within five minutes. The MAN background technique proves to be very helpful in the case of REE-analysis (L-lines on $\mathrm{LiF}$ ), as a suitable background point is difficult to find. This setting yields detection limits for all REE around 300-1000 ppm at $20 \mathrm{nA}$, and permits a quick identification of complex REE-minerals. For higher precision, a second setup focusing on $\mathrm{U}-\mathrm{Th}-\mathrm{Pb}$ was prepared using a high current (200 nA) and reserving three spectrometers for $\mathrm{U}$, Th and $\mathrm{Pb}$ (with TDI correction). Accuracy of the background correction on $\mathrm{U}$, Th and $\mathrm{Pb}$ was ensured using the multipoint background technique [7]. After 10 minutes counting, single-point detection limits around 100 ppm for $\mathrm{U}$ and $\mathrm{Th}$ and $30 \mathrm{ppm}$ for $\mathrm{Pb}$ was reached, and analysis of secondary standards yields adequate age results. Unfortunately, low analytical totals around 96-98\% suggest beam damage. To prevent this, metal coating is required. To further improve the analytical efficiency, new protocol to be developed might include a two-step analysis: (1) fast REE analysis at low current to prevent beam damage, possibly combining EDS data to further reduce the analysis time, and (2) a high current analysis of $\mathrm{U}, \mathrm{Th}, \mathrm{Pb}$ and some other crucial (interfering or minor/trace) elements with a TDI correction to compensate for beam damage.

\section{References:}

[1] R Castaing doctoral thesis, Université de Paris, (1951) Publication ONERA N. 55.

[2] J Fournelle \& PK Carpenter Microscopy \& Microanalysis 12 (S02), (2006), p. 880.

[3] PK Carpenter EPMA Topical Conference, Madison (WI, USA) (2016).

[4] JM Allaz \& PK Carpenter EPMA Topical Conference, Madison (WI, USA) (2016).

[5] P Hovington et al, Journal of Scanning Microscopies 1, (1997), p. 1.

[6] JJ Donovan \& TN Tingle, Journal of Microscopy and Microanalyis 2, (1996), p. 1.

[7] JM Allaz et al, Microscopy \& Microanalysis 20 (S03), (2014), p. 720. 